\title{
The geometry behind double geometry
}

\author{
Martin Cederwall \\ Dept. of Fundamental Physics, Chalmers University of Technology, \\ SE 412 96 Gothenburg, Sweden \\ E-mail: martin.cederwall@chalmers.se
}

ABstract: Generalised diffeomorphisms in double field theory rely on an $O(d, d)$ structure defined on tangent space. We show that any (pseudo-)Riemannian metric on the doubled space defines such a structure, in the sense that the generalised diffeomorphisms defined using such a metric form an algebra, provided a covariant section condition is fulfilled. Consistent solutions of the section condition gives further restrictions. The case previously considered corresponds to a flat metric. The construction makes it possible to apply double geometry to a larger class of manifolds. Examples of curved defining metrics are given. We also comment on the rôle of the defining geometry for the symmetries of double field theory, and on the continuation of the present construction to the U-duality setting.

Keywords: Differential and Algebraic Geometry, Space-Time Symmetries, String Duality

ARXIV EPRINT: 1402.2513 
Generalised geometry (see refs. [1-38]) has arisen as a means to geometrise duality symmetries. By using extended space-times, reduced to the physical ones by a section condition, the local symmetries of gravity and tensor fields unite in the generalised diffeomorphisms. This permits not only a more symmetric formulation of the massless degrees of string theory or M-theory, but also a situation where, for certain backgrounds, duality symmetries are understood from a manifestly geometric perspective, and where it is possible to move beyond strictly geometric backgrounds.

Recent work [39-43] has shed more light on global questions of generalised geometry. In particular, the global questions about the structure of extended manifolds have been asked and partially answered, although there are remaining issues. This is closely related to the original main purpose of the programme, namely to make the appearance of duality symmetries as manifest as possible. It is important to note that these should not be built into the formalism as global symmetries, but rather arise as "generalised isometries", special symmetries arising in special (e.g. toroidal) backgrounds, just as isometries arise in in ordinary geometry. It is by now known $[7,8,41,43]$ that the full duality group can not be obtained this way, at least not with the present formalism and level of understanding. As we will see, one way forward may be to include ordinary diffeomorphisms.

The extended manifolds have however so far been restricted to manifolds equipped with a globally defined flat metric. This is because the definition of the generalised diffeomorphism transformations involves such a flat metric $\eta_{M N}$. This metric defines an $O(d, d)$ structure on the extended space. In the following, we will examine to what extent the defining metric can be chosen differently. If one wants to consider not only generalised diffeomorphisms (under which the defining metric is invariant), but also ordinary diffeomorphisms, this becomes necessary, already for the case of the flat metric.

The usual definition of the double diffeomorphisms contains a parameter $\xi^{M}$ on the doubled space. Fields transform under double diffeomorphisms so that, in addition to the translation generated by $\xi=\xi^{M} \partial_{M}$, they are rotated by an $s o(d, d)$ transformation generated by $a-a^{t}$, where $a_{M}^{N}=\partial_{M} \xi^{N}$. Here, the transpose is defined using a constant metric $\eta_{M N}$, invariant under $O(d, d) \subset G L(2 d)$, such that $\left(a^{t}\right)_{M^{N}}=\eta_{M P} \eta^{N Q} \partial_{Q} \xi^{P}$. Acting on a covector (which is equivalent to a vector, using $\eta$ ), the standard form of a double diffeomorphism thus becomes

$$
\mathcal{L}_{\xi} V=\left(\xi+a-a^{t}\right) V=\left(L_{\xi}-a^{t}\right) V .
$$

It is then straightforward to verify that, when all fields, including the transformation parameters, obey the section condition

$$
\eta^{M N} \partial_{M} \otimes \partial_{N}=0
$$

(the " $\otimes "$ notation meaning that the two derivative may act on the same field or any pair), the commutator of two double diffeomorphisms is again a double diffeomorphism:

$$
\begin{aligned}
{\left[\mathcal{L}_{\xi}, \mathcal{L}_{\eta}\right] } & =\mathcal{L}_{\llbracket \xi, \eta \rrbracket}, \\
\text { where } \quad \llbracket \xi, \eta \rrbracket & =\frac{1}{2}\left(\mathcal{L}_{\xi} \eta-\mathcal{L}_{\eta} \xi\right) .
\end{aligned}
$$


In ref. [41], this was elucidated by the observation that

$$
\llbracket \xi, \eta \rrbracket=[\xi, \eta]+\chi_{\xi, \eta},
$$

where $\chi_{\xi, \eta}^{M}=\frac{1}{2}\left(-\xi^{N} \partial^{M} \eta_{N}+\eta^{N} \partial^{M} \xi_{N}\right)$ is a non-translating parameter, i.e., one for which $\chi^{M} \partial_{M}=0$ using the section condition. Therefore, $\mathcal{L}_{\chi_{\xi, \eta}}=\Delta_{\xi, \eta}$ is a specific local $s o(d, d)$ transformation, which also turns out to be nilpotent. Using also $b_{M}{ }^{N}=\partial_{M} \eta^{N}$, the explicit form of $\Delta$ is

$$
\Delta_{\xi, \eta}=-a b^{t}+b a^{t} .
$$

The commutator can thus also be written

$$
\left[\mathcal{L}_{\xi}, \mathcal{L}_{\eta}\right]=\mathcal{L}_{[\xi, \eta]}+\Delta_{\xi, \eta}
$$

This observation was used in ref. [41] to explain the abelian gerbe structure encoded in double diffeomorphisms.

We thus observe that the double diffeomorphisms rely on the existence of a flat metric $\eta_{M N}$. This implies no restriction locally, but limits the choice of double manifolds to those globally allowing such a metric structure. ${ }^{1}$ These of course include tori, which are of special interest since they lead to the ordinary (discrete) T-duality. It seems to be of no immediate interest to introduce curvature locally, since the defining metric is non-dynamical, but it may be important to be able to include topologies that demand a non-flat metric, or indeed also in the flat situation. This is the main subject of this paper.

A local $O(d, d)$ structure is induced by any metric $H_{M N}$. We therefore ask the questions: To what extent is it possible to use a (non-flat) metric $H$ on the double space? What are the restrictions on such a metric implied by the existence of an algebra of double diffeomorphisms? As we will see, any (pseudo-)Riemannian metric (of signature $(d, d)$ ) is algebraically allowed, and further restriction follow only from the existence of solutions to the section condition.

We would like to stress that the introduction of the metric $H$ has nothing to do with equipping the double manifold with a generalised metric, containing the metric and $B$-field on a subspace obtained by solving the section condition. The metric $H$ is thought of as an ordinary metric on the double space, whose purpose is to define a local $O(d, d)$ structure. It will define an ordinary torsion-free affine connection $\Gamma$ and a Riemann tensor $R$.

The Ansatz we will use for the double diffeomorphisms is the natural one that reduces to eq. (1) when $H=\eta$. In order to obtain covariance, we should then use covariant derivatives $D$, containing $\Gamma$, throughout, and use the covariantly constant metric $H$ to raise and lower indices (i.e., to define the transpose of a matrix). Consider therefore a transformation defined as

$$
\mathcal{L}_{\xi} V_{M}=\xi^{N} D_{N} V_{M}+\left(a-a^{t}\right)_{M}{ }^{N} V_{N}=\left(L_{\xi}-a^{t}\right)_{M}{ }^{N} V_{N},
$$

where $a_{M}{ }^{N}=D_{M} \xi^{N}$ and $\left(a^{t}\right)_{M^{N}}=H_{M P} H^{N Q} a_{Q}{ }^{P}$. As usual, the connection terms in the Lie derivative cancel, and the choice of defining metric is only reflected in the last term. It

\footnotetext{
${ }^{1}$ This of course only implies if one insists on the possibility of applying ordinary diffeomorphisms.
} 
is clear that $H$ itself is conserved by such a transformation (extended to tensors), since it is covariantly constant and

$$
\mathcal{L}_{\xi} H_{M N}=2\left(a-a^{t}\right){ }_{\left(M^{P}\right.} H_{N) P}=0 .
$$

In order to check the algebra of these generalised diffeomorphisms, we also need a section condition, which will be the natural generalisation of eq. (2), namely

$$
H^{M N} D_{M} \otimes D_{N}=0
$$

Note that there is no need of a section condition involving the metric, since it is covariantly constant.

Let us now commute two transformations of the type (7). Noting that

$$
\left[\mathcal{L}_{\xi}, \mathcal{L}_{\eta}\right]=\left[L_{\xi}-a^{t}, L_{\eta}-b^{t}\right]
$$

(where of course also $b$ is defined with the covariant derivative, $b_{M}{ }^{N}=D_{M} \eta^{N}$ ), it becomes clear that any obstruction containing curvature will reside in terms containing $a^{t}$ or $b^{t}$. A careful calculation, now keeping track of the order of covariant derivatives, yields

$$
\begin{aligned}
& \left(\left[\mathcal{L}_{\xi}, \mathcal{L}_{\eta}\right]-\mathcal{L}_{[\xi, \eta]}\right)_{M}{ }^{N} \\
& \quad=-\left[a, b^{t}\right]_{M}{ }^{N}+\left[b, a^{t}\right]_{M}{ }^{N}+\xi^{P}\left[D^{N}, D_{P}\right] \eta_{M}-\eta^{P}\left[D^{N}, D_{P}\right] \xi_{M} \\
& \quad=\left(-a b^{t}+b a^{t}\right)_{M}{ }^{N}+2 R^{N}{ }_{P M Q} \xi^{[P} \eta^{Q]}
\end{aligned}
$$

In the second step, the section condition has been used in the form $a^{t} b=0$ etc. The question now is whether this remainder can be written as a non-translating transformation $\mathcal{L}_{\chi}$ as in the case of flat metric. Consider a parameter $\chi_{\xi, \eta}^{M}=\frac{1}{2}\left(-\xi^{N} D^{M} \eta_{N}+\eta^{N} D^{M} \xi_{N}\right)$. Then

$$
\begin{aligned}
\left(\Delta_{\xi, \eta}\right)_{M}{ }^{N}=\left(\mathcal{L}_{\chi}\right)_{M}{ }^{N} & =\left(-a b^{t}+b a^{t}\right)_{M^{N}}-\frac{1}{2} \xi^{P}\left[D_{M}, D^{N}\right] \eta_{P}+\frac{1}{2} \eta^{P}\left[D_{M}, D^{N}\right] \xi_{P} \\
& =\left(-a b^{t}+b a^{t}\right)_{M^{N}}-R_{M}{ }^{N}{ }_{P Q} \xi^{P} \eta^{Q}
\end{aligned}
$$

Comparing eqs. (11) and (12), we see that they are equal modulo the (vanishing) torsion Bianchi identity $R_{[P Q M]}{ }^{N}=0$.

This shows the somewhat surprising result that there is no curvature obstruction to the existence of an algebra of double diffeomorphisms. The metric $H$ defining the local $O(d, d)$ structure can be taken as any (pseudo-)Riemannian metric. The commutator is still formally given by eq. (3) or eq. (6), although the definition of the bracket $\llbracket \cdot, \cdot \rrbracket$ is metric-dependent, so our new algebras are most likely non-isomorphic to the flat one.

Once we have established the formal closure of the algebra (strictly speaking, algebroid) of double diffeomorphisms with the defining metric $H$, it is important also to investigate possible solutions of the section condition. Solving the section condition amounts to finding a $d$-dimensional isotropic subspace of tangent space, spanned by the $\tilde{m}$ directions in a split $X^{M}=\left(x^{m}, y^{\tilde{m}}\right)$, such that $D_{\tilde{m}}=0$ on all fields. Acting with further covariant derivatives gives the integrability condition $R_{\tilde{m} N P}{ }^{Q}=0$. If this condition is fulfilled there will be a 
class of choices of coordinates where $D_{\tilde{m}}=\partial_{\tilde{m}}$. Examples of such metrics are given by the pp-wave-like space-times

$$
d s^{2}=H_{M N} d X^{M} d X^{N}=h_{m n}(x) d x^{m} d x^{n}+2 \delta_{m \tilde{m}} d x^{m} d y^{\tilde{m}},
$$

with a set of $d$ light-like Killing vectors $\partial_{\tilde{m}}$. It is not obvious to us whether this provides an exhaustive list of allowed defining metrics. In any case, this class is general enough to give room for any topology of a physical compactification space (with coordinates $x^{m}$ ).

There should be analogous structures in exceptional extended geometry. Unlike the case of doubled space, both the generalised diffeomorphisms and the section condition involve a structure which is not a metric, but a tensor defining an $E_{n(n)} \times \mathbb{R}^{+}$structure, the so called $Y$ tensor [29]. The transformations look formally the same as in eq. (1), but with $a^{t}$ replaced by $a^{Y}$, with components $\left(a^{Y}\right)_{M^{N}}=Y_{M P}{ }^{Q N} a_{Q}{ }^{P}$, and the "flat" section condition reads $Y_{M N}{ }^{P Q} \partial_{P} \otimes \partial_{Q}=0$. The $Y$ tensor does not factorise into a product of a metric and its inverse, so the structure sought for is not a metric structure. Nevertheless, it should be possible to pursue a similar investigation in these cases.

We would finally like to comment on the symmetries of double geometry (the remarks apply to extended geometry in general). It is known that the generalised diffeomorphisms are not general enough to accommodate overlaps that would give truly non-geometric solutions $[7,8,41,43]$. This is because of the section condition. Once a solution to the section condition is chosen, it is preserved by the generalised diffeomorphisms, which effectively prevents some duality transformations, namely those that would act on the extended space in way that changes the solution to the section condition (the section condition itself is of course preserved). Therefore, the whole (discrete) T-duality group of some compactification can not, with the present understanding, be constructed as generalised isometries. (This situation is by no means improved by the construction of the present paper, rather the opposite, since the possibilities of changing the section condition tend to be fewer.)

A proper understanding of the section condition, and of possible ways to relax it (see e.g. refs. [44-47]) is thus one of the key problems in extended geometry. A final solution to this problem will probably have to await a formulation where the section condition is not applied "by hand", but arises dynamically, as does the string theory level matching condition. In the meantime, it is reasonable to expect that in such a formulation, although the defining metric (or exceptional structure) is not dynamical, its symmetries, which in the case of double field theory are the isometries of $H$, can be included as gauge symmetries. If this is the case, this will suffice to fill out the T-duality group. We note that, while ordinary diffeomorphisms in general do not have good commutators with the generalised diffeomorphisms $\mathcal{L}_{\xi}$, since they change the defining metric, isometries do. If $u^{M}$ is a Killing vector of $H$ it is straightforward to check that $\left[L_{u}, \mathcal{L}_{\xi}\right]=\mathcal{L}_{[u, \xi]}$ (the analogous statement for a finite isometry is obviously true as well). Here, $u^{M}$ does not need to obey the solution to the section condition, and it is only Killing vectors not obeying it that generate transformations not contained in the $\mathcal{L}$ 's. Hopefully, this way of constructing the full duality group can be a first step in resolving the dilemma of obtaining duality symmetries from extended field theories. 


\section{Acknowledgments}

The author would like to thank David Berman, Anna Karlsson, George Papadopoulos and Malcolm Perry for discussions.

Open Access. This article is distributed under the terms of the Creative Commons Attribution License (CC-BY 4.0), which permits any use, distribution and reproduction in any medium, provided the original author(s) and source are credited.

\section{References}

[1] C.M. Hull, A geometry for non-geometric string backgrounds, JHEP 10 (2005) 065 [hep-th/0406102] [INSPIRE].

[2] C.M. Hull, Doubled geometry and T-folds, JHEP 07 (2007) 080 [hep-th/0605149] [INSPIRE].

[3] N.J. Hitchin, Lectures on special Lagrangian submanifolds, math.DG/9907034 [INSPIRE].

[4] N. Hitchin, Lectures on generalized geometry, arXiv:1008.0973 [INSPIRE].

[5] W. Siegel, Two vierbein formalism for string inspired axionic gravity, Phys. Rev. D 47 (1993) 5453 [hep-th/9302036] [INSPIRE].

[6] C.M. Hull, Generalised geometry for M-theory, JHEP 07 (2007) 079 [hep-th/0701203] [INSPIRE].

[7] C. Hull and B. Zwiebach, The gauge algebra of double field theory and Courant brackets, JHEP 09 (2009) 090 [arXiv:0908.1792] [INSPIRE].

[8] C. Hull and B. Zwiebach, Double field theory, JHEP 09 (2009) 099 [arXiv:0904.4664] [INSPIRE].

[9] O. Hohm, C. Hull and B. Zwiebach, Background independent action for double field theory, JHEP 07 (2010) 016 [arXiv: 1003.5027] [INSPIRE].

[10] O. Hohm, C. Hull and B. Zwiebach, Generalized metric formulation of double field theory, JHEP 08 (2010) 008 [arXiv: 1006.4823] [INSPIRE].

[11] O. Hohm and B. Zwiebach, On the Riemann tensor in double field theory, JHEP 05 (2012) 126 [arXiv:1112.5296] [INSPIRE].

[12] O. Hohm and B. Zwiebach, Towards an invariant geometry of double field theory, J. Math. Phys. 54 (2013) 032303 [arXiv:1212.1736] [INSPIRE].

[13] I. Jeon, K. Lee and J.-H. Park, Ramond-Ramond cohomology and $O_{D, D}$ T-duality, JHEP 09 (2012) 079 [arXiv: 1206.3478] [INSPIRE].

[14] I. Jeon, K. Lee and J.-H. Park, Differential geometry with a projection: application to double field theory, JHEP 04 (2011) 014 [arXiv: 1011.1324] [INSPIRE].

[15] I. Jeon, K. Lee and J.-H. Park, Stringy differential geometry, beyond Riemann, Phys. Rev. D 84 (2011) 044022 [arXiv:1105.6294] [InSPIRE].

[16] I. Jeon, K. Lee and J.-H. Park, Supersymmetric double field theory: stringy reformulation of supergravity, Phys. Rev. D 85 (2012) 081501 [Erratum ibid. D 86 (2012) 089903] [arXiv:1112.0069] [INSPIRE]. 
[17] O. Hohm and S.K. Kwak, $N=1$ supersymmetric double field theory, JHEP 03 (2012) 080 [arXiv:1111.7293] [INSPIRE].

[18] O. Hohm and S.K. Kwak, Frame-like geometry of double field theory, J. Phys. A 44 (2011) 085404 [arXiv:1011.4101] [inSPIRE].

[19] O. Hohm, S.K. Kwak and B. Zwiebach, Unification of type II strings and T-duality, Phys. Rev. Lett. 107 (2011) 171603 [arXiv:1106.5452] [INSPIRE].

[20] O. Hohm, S.K. Kwak and B. Zwiebach, Double field theory of type II strings, JHEP 09 (2011) 013 [arXiv: 1107.0008] [INSPIRE].

[21] K. Lee and J.-H. Park, Covariant action for a string in "doubled yet gauged" spacetime, Nucl. Phys. B 880 (2014) 134 [arXiv:1307.8377] [INSPIRE].

[22] D.S. Berman, C.D.A. Blair, E. Malek and M.J. Perry, The $O_{D, D}$ geometry of string theory, Int. J. Mod. Phys. A 29 (2014) 1450080 [arXiv:1303.6727] [InSPIRE].

[23] D.S. Berman and M.J. Perry, Generalized geometry and M-theory, JHEP 06 (2011) 074 [arXiv: 1008.1763] [INSPIRE].

[24] D.S. Berman, H. Godazgar and M.J. Perry, $\mathrm{SO}(5,5)$ duality in M-theory and generalized geometry, Phys. Lett. B $\mathbf{7 0 0}$ (2011) 65 [arXiv:1103.5733] [INSPIRE].

[25] D.S. Berman, H. Godazgar, M. Godazgar and M.J. Perry, The local symmetries of M-theory and their formulation in generalised geometry, JHEP 01 (2012) 012 [arXiv:1110.3930] [INSPIRE].

[26] D.S. Berman, H. Godazgar, M.J. Perry and P. West, Duality invariant actions and generalised geometry, JHEP 02 (2012) 108 [arXiv:1111.0459] [INSPIRE].

[27] A. Coimbra, C. Strickland-Constable and D. Waldram, $E_{d(d)} \times \mathbb{R}^{+}$generalised geometry, connections and M-theory, JHEP 02 (2014) 054 [arXiv:1112.3989] [INSPIRE].

[28] A. Coimbra, C. Strickland-Constable and D. Waldram, Supergravity as generalised geometry II: $E_{d(d)} \times \mathbb{R}^{+}$and M-theory, JHEP 03 (2014) 019 [arXiv: 1212.1586] [INSPIRE].

[29] D.S. Berman, M. Cederwall, A. Kleinschmidt and D.C. Thompson, The gauge structure of generalised diffeomorphisms, JHEP 01 (2013) 064 [arXiv:1208.5884] [INSPIRE].

[30] J.-H. Park and Y. Suh, U-geometry: SL(5), JHEP 04 (2013) 147 [Erratum ibid. 11 (2013) 210] [arXiv: 1302.1652] [INSPIRE].

[31] M. Cederwall, J. Edlund and A. Karlsson, Exceptional geometry and tensor fields, JHEP 07 (2013) 028 [arXiv:1302.6736] [INSPIRE].

[32] M. Cederwall, Non-gravitational exceptional supermultiplets, JHEP 07 (2013) 025 [arXiv: 1302.6737] [INSPIRE].

[33] G. Aldazabal, M. Graña, D. Marqués and J.A. Rosabal, Extended geometry and gauged maximal supergravity, JHEP 06 (2013) 046 [arXiv: 1302.5419] [INSPIRE].

[34] O. Hohm and H. Samtleben, Exceptional field theory I: $E_{6(6)}$ covariant Form of M-theory and type IIB, Phys. Rev. D 89 (2014) 066016 [arXiv:1312.0614] [INSPIRE].

[35] O. Hohm and H. Samtleben, Exceptional field theory II: $E_{7(7)}$, Phys. Rev. D 89 (2014) 066017 [arXiv:1312.4542] [INSPIRE].

[36] G. Aldazabal, M. Graña, D. Marqués and J.A. Rosabal, The gauge structure of exceptional field theories and the tensor hierarchy, JHEP 04 (2014) 049 [arXiv: 1312.4549] [INSPIRE]. 
[37] C.D.A. Blair, E. Malek and J.-H. Park, M-theory and type IIB from a duality manifest action, JHEP 01 (2014) 172 [arXiv:1311.5109] [INSPIRE].

[38] C. Strickland-Constable, Subsectors, Dynkin diagrams and new generalised geometries, arXiv: 1310.4196 [INSPIRE].

[39] O. Hohm and B. Zwiebach, Large gauge transformations in double field theory, JHEP 02 (2013) 075 [arXiv: 1207.4198] [INSPIRE].

[40] J.-H. Park, Comments on double field theory and diffeomorphisms, JHEP 06 (2013) 098 [arXiv: 1304.5946] [INSPIRE].

[41] D.S. Berman, M. Cederwall and M.J. Perry, Global aspects of double geometry, arXiv: 1401.1311 [INSPIRE].

[42] O. Hohm, D. Lüst and B. Zwiebach, The spacetime of double field theory: review, remarks and outlook, Fortsch. Phys. 61 (2013) 926 [arXiv:1309.2977] [INSPIRE].

[43] G. Papadopoulos, Seeking the balance: patching double and exceptional field theories, arXiv: 1402.2586 [INSPIRE].

[44] D. Geissbuhler, Double field theory and $N=4$ gauged supergravity, JHEP 11 (2011) 116 [arXiv: 1109.4280] [INSPIRE].

[45] G. Aldazabal, W. Baron, D. Marques and C. Núñez, The effective action of double field theory, JHEP 11 (2011) 052 [Erratum ibid. 11 (2011) 109] [arXiv:1109.0290] [INSPIRE].

[46] D.S. Berman, E.T. Musaev, D.C. Thompson and D.C. Thompson, Duality invariant M-theory: gauged supergravities and Scherk-Schwarz reductions, JHEP 10 (2012) 174 [arXiv: 1208.0020] [INSPIRE].

[47] A. Betz, R. Blumenhagen, D. Lüst and F. Rennecke, A note on the CFT origin of the strong constraint of DFT, JHEP 05 (2014) 044 [arXiv: 1402.1686] [INSPIRE]. 